\title{
Particle physics model of curvaton inflation in a stable universe
}

\author{
Zoltán Péli ${ }^{*}$ and István Nándori ${ }^{\dagger}$ \\ MTA-DE Particle Physics Research Group, H-4010 Debrecen, PO Box 105, Hungary \\ Zoltán Trócsányi $\oplus^{\ddagger}$ \\ Institute for Theoretical Physics, ELTE Eötvös Loránd University, Pázmány Péter sétány 1/A, \\ H-1117 Budapest, Hungary \\ and MTA-DE Particle Physics Research Group, H-4010 Debrecen, PO Box 105, Hungary
}

(Received 26 November 2019; revised manuscript received 17 February 2020; accepted 6 March 2020; published 27 March 2020)

\begin{abstract}
We investigate a particle physics model for cosmic inflation based on the following assumptions: (i) there are at least two complex scalar fields; (ii) the scalar potential is bounded from below and remains perturbative up to the Planck scale; (iii) we assume slow-roll inflation with maximally correlated adiabatic and entropy fluctuations 50-60 e-folds before the end of inflation. The energy scale of the inflation is set automatically by the model. Assuming also at least one massive right-handed neutrino, we explore the allowed parameter space of the scalar potential as a function of the Yukawa coupling of this neutrino.
\end{abstract}

DOI: $10.1103 /$ PhysRevD.101.063533

\section{INTRODUCTION}

The standard model (SM) of elementary particle interactions [1] has been proven experimentally to high precision at the Large Electron Positrion Collider [2] and also at the Large Hadron Collider (LHC) $[3,4]$. At the LHC the last missing piece, the Higgs particle has also been discovered and its mass has been measured at high precision [5,6], which made possible the precise renormalization group (RG) flow analysis of the Brout-EnglertHiggs potential $[7,8]$. The perturbative precision of this computation is sufficiently high so that the conclusion about the instability of the vacuum in the standard model cannot be questioned. While the instability may not influence the fate of our present Universe if the tunneling rate from the false vacuum is sufficiently low (making the Universe metastable), one may insist that the vacuum must be stable up to the Planck scale. Indeed, if we assume that the characteristic energy scale of particle interactions were close to the Planck scale immediately after the big bang, the Universe based on the SM was unstable and could not exist, which calls for an extension of the SM. Presently it is widely accepted that such an extension should explain not

\footnotetext{
*zoltanpeli92@gmail.com

†nandori.istvan@science.unideb.hu

*Zoltan.Trocsanyi@cern.ch http://pppheno.elte.hu/
}

Published by the American Physical Society under the terms of the Creative Commons Attribution 4.0 International license. Further distribution of this work must maintain attribution to the author(s) and the published article's title, journal citation, and DOI. Funded by SCOAP. only open questions in particle physics—such as the origin of neutrino masses-but also those in cosmology.

One of the vigorously studied questions in our understanding of the early universe is the physics of cosmic inflation. Today the original idea for inflation [9] is not favored because it is unclear how to define a proper mechanism to explain the required reheating of the universe. A popular solution to this question of reheating is the slow-roll scenario $[10,11]$ in which the ground state starts from an unstable position and rolls down very slowly to a local or global minimum. The inflation stops when the potential energy function becomes too steep, which leads to a fast roll. In principle, the slow roll can start from a large field value and proceed toward a minimum with a smaller field value, or from a small (essentially vanishing) field value to a larger minimum. These two cases are referred to as large- and small-field slow roll [12]. A problem related to large-field slow-roll is the initial value problem, namely one has to explain why the ground state starts from a value much larger than the typical energy scale of inflation. Chaotic inflation [13] was devised to handle this problem, but then one has to assume very large-again larger than the scale of inflation-fluctuations. The origin of inflation and especially the emergence of the inflationary potential is still an open question in cosmology $[14,15]$.

It is known that scalar fields can mimic the equation of state required for the exponential expansion of the early universe [10,11]. As the Higgs boson was discovered, we know that at least one doublet scalar field exists in nature. Hence, it may appear natural to assume that the BroutEnglert-Higgs (BEH) field is the inflaton (see for example Ref. [16]), but such a scenario was criticized, see for instance Ref. [17]. Many types of scalar potentials have 
already been discussed in the literature as viable scenarios for cosmic inflation [17]. There are three major categories of scalar inflaton potentials with minimal kinetic terms: (i) the large field, (ii) the small field and (iii) the hybrid models. In the third case one introduces more than one field, with one of those being the inflaton and the other field switches off the exponential expansion. In this sense it is not a real multifield model. The case of hybrid models is excluded by experimental observations because those predict a scalar tilt $n_{s}$ larger than one in contradiction with the observed structure of the thermal fluctuations of the cosmic microwave background radiation (CMBR) resulting in $n_{s}=0.9677 \pm 0.0060[18,19]$. The tensor and scalar power spectra of the CMBR suggest a small value for the tensor-to-scalar ratio $r$, consistent with zero, which emerges automatically in real multifield models with curvaton scenario [20-23].

Here we consider the simplest possible extension of the SM that has the potential to explain the emergence of the inflationary potential. The complete particle physics model of this new superweak force was published in Ref. [24]. We study the renormalization group flow of the scalar couplings of this extension of the SM at one-loop order in perturbation theory. We find that for small values of the new gauge couplings-as suggested by other phenomenological considerations - the only relevant couplings are the scalar ones and the largest Yukawacoupling in the neutrino sector if we assume similar hierarchy of the latter as one can observe for u-type quarks in the SM [25]. Hence, the precise formulation of the gauge sector does not influence our conclusions, so in this article we present only the scalar and relevant Yukawa sector in detail. We also find that the extended scalar sector can serve as a simple multifield model of cosmic inflation with a curvaton scenario. We show that in a fairly constrained region of the parameter space, the model can provide a natural switch on and off mechanism of inflation.

\section{PARTICLE PHYSICS MODEL}

The particle content of the model coincides with that in the standard model of particle interactions, supplemented with one complex scalar field and three right-handed neutrinos. The latter can be either Dirac- or Majoranatype. While we present the relevant renormalization group equations (RGE) for both cases, for the sake of definiteness we present physical predictions only for the case of Dirac neutrinos.

\section{A. Scalar sector}

Our scalar sector is defined similarly as in the SM, but in addition to the usual scalar field $\phi$ that is an $S U(2)$-doublet

$$
\phi=\left(\begin{array}{c}
\phi^{+} \\
\phi^{0}
\end{array}\right)=\frac{1}{\sqrt{2}}\left(\begin{array}{c}
\phi_{1}+\mathrm{i} \phi_{2} \\
\phi_{3}+\mathrm{i} \phi_{4}
\end{array}\right)
$$

we assume the existence of a complex scalar $\chi$ that transforms as a singlet under the SM gauge transformations. The gauge invariant Lagrangian of the scalar fields is

$\mathcal{L}_{\phi, \chi}=\left[D_{\mu}^{(\phi)} \phi\right]^{*} D^{(\phi) \mu} \phi+\left[D_{\mu}^{(\chi)} \chi\right]^{*} D^{(\chi) \mu} \chi-V(\phi, \chi)$.

The covariant derivative for the new scalar $\chi$ is

$$
D_{\mu}^{(\chi)}=\partial_{\mu}-\mathrm{i} g_{Z} Z_{\mu}
$$

where $Z_{\mu}$ is the new gauge field and $g_{Z}$ is the new gauge coupling. In the renormalization group analysis below we shall concentrate on the phenomenologically relevant case when the new couplings are superweak $g_{Z} \ll 1$, hence negligible in the scalar sector.

In Eq. (2) the potential energy is defined as

$V(\phi, \chi)=V_{0}-\mu_{\phi}^{2}|\phi|^{2}-\mu_{\chi}^{2}|\chi|^{2}+\frac{1}{2}\left(|\phi|^{2},|\chi|^{2}\right) C\left(\begin{array}{c}|\phi|^{2} \\ |\chi|^{2}\end{array}\right)$

where $|\phi|^{2}=\left|\phi^{+}\right|^{2}+\left|\phi^{0}\right|^{2}$ and

$$
C=\left(\begin{array}{cc}
2 \lambda_{\phi} & \lambda \\
\lambda & 2 \lambda_{\chi}
\end{array}\right)
$$

is the coupling matrix. This potential energy function contains a coupling term $\lambda|\phi|^{2}|\chi|^{2}$ of the scalar fields in addition to the usual quartic terms. The value of the additive constant $V_{0}$ is irrelevant for particle dynamics, but as we shall see, it is relevant for the inflationary model, hence we allow a nonvanishing value for it. In order that this potential energy be bounded from below, we have to require the positivity of the self-couplings, $\lambda_{\phi}, \lambda_{\chi}>0$. The eigenvalues of the coupling matrix are

$$
\lambda_{ \pm}=\frac{1}{2}\left(\lambda_{\phi}+\lambda_{\chi} \pm \sqrt{\left(\lambda_{\phi}-\lambda_{\chi}\right)^{2}+\lambda^{2}}\right),
$$

with $\lambda_{+}>0$ and $\lambda_{-}<\lambda_{+}$. In the physical region the potential can be unbounded from below only if $\lambda_{-}<0$ and the eigenvector belonging to $\lambda_{-}$points into the first quadrant, which may occur only when $\lambda<0$. In this case, the potential will be bounded from below if the coupling matrix is positive definite, i.e.,

$$
\operatorname{det} C=4 \lambda_{\phi} \lambda_{\chi}-\lambda^{2}>0 .
$$

If these conditions are satisfied, we find the minimum of the potential energy at field values $\phi=v / \sqrt{2}$ and $\chi=w / \sqrt{2}$ where the vacuum expectation values (VEVs) are 
TABLE I. Possible signs of the couplings in the scalar potential $V(\phi, \chi)$ in order to have two nonvanishing real VEVs. $\Theta$ is the step function, $\Theta(x)=1$ if $x>0$ and 0 if $x<0$.

\begin{tabular}{lccccc}
\hline \hline$\Theta(\lambda)$ & $\Theta\left(\lambda_{\phi}\right)$ & $\Theta\left(\lambda_{\chi}\right)$ & $\Theta\left(4 \lambda_{\phi} \lambda_{\chi}-\lambda^{2}\right)$ & $\Theta\left(\mu_{\phi}^{2}\right) \Theta\left(\mu_{\chi}^{2}\right)$ & $\Theta\left(2 \lambda_{\chi} \mu_{\phi}^{2}-\lambda \mu_{\chi}^{2}\right) \Theta\left(2 \lambda_{\phi} \mu_{\chi}^{2}-\lambda \mu_{\phi}^{2}\right)$ \\
\hline 1 & 1 & 1 & Unconstrained & 1 & Unconstrained \\
0 & 1 & 1 & 1 & 1 & Unconstrained \\
0 & 1 & 1 & 1 & 0 & 1 \\
\hline \hline
\end{tabular}

$v=\sqrt{2} \sqrt{\frac{2 \lambda_{\chi} \mu_{\phi}^{2}-\lambda \mu_{\chi}^{2}}{4 \lambda_{\phi} \lambda_{\chi}-\lambda^{2}}}, \quad w=\sqrt{2} \sqrt{\frac{2 \lambda_{\phi} \mu_{\chi}^{2}-\lambda \mu_{\phi}^{2}}{4 \lambda_{\phi} \lambda_{\chi}-\lambda^{2}}}$.

Using the VEVs, we can express the quadratic couplings as

$$
\mu_{\phi}^{2}=\lambda_{\phi} v^{2}+\frac{\lambda}{2} w^{2}, \quad \mu_{\chi}^{2}=\lambda_{\chi} w^{2}+\frac{\lambda}{2} v^{2},
$$

so those are both positive if $\lambda>0$. If $\lambda<0$, the constraint (7) ensures that the denominators of the VEVs in Eq. (8) are positive, so the VEVs have nonvanishing real values only if

$$
2 \lambda_{\chi} \mu_{\phi}^{2}-\lambda \mu_{\chi}^{2}>0 \quad \text { and } 2 \lambda_{\phi} \mu_{\chi}^{2}-\lambda \mu_{\phi}^{2}>0
$$

simultaneously, which can be satisfied if at most one of the quadratic couplings is smaller than zero. We summarize the possible cases for the signs of the couplings in Table I.

After spontaneous symmetry breaking, we use the following convenient parametrization for the scalar fields:

$$
\phi=\frac{1}{\sqrt{2}} \mathrm{e}^{\mathrm{i} T \cdot \xi(x) / v}\left(\begin{array}{c}
0 \\
v+h^{\prime}(x)
\end{array}\right)
$$

and

$$
\chi(x)=\frac{1}{\sqrt{2}} \mathrm{e}^{\mathrm{i} \eta(x) / w}\left(w+s^{\prime}(x)\right),
$$

with $\boldsymbol{T}=\left(T_{1}, T_{2}, T_{3}\right)$ denoting the generators of the SU(2) group, $\boldsymbol{\xi}=\left(\xi_{1}, \xi_{2}, \xi_{3}\right)$ and $\eta$ being real functions. We can use the gauge invariance of the model to choose the unitary gauge when

$\phi^{\prime}(x)=\frac{1}{\sqrt{2}}\left(\begin{array}{c}0 \\ v+h^{\prime}(x)\end{array}\right)$ and $\chi^{\prime}(x)=\frac{1}{\sqrt{2}}\left(w+s^{\prime}(x)\right)$.

We can diagonalize the mass matrix (quadratic terms) of the two real scalars $\left(h^{\prime}\right.$ and $\left.s^{\prime}\right)$ by the rotation

$$
\left(\begin{array}{l}
h \\
s
\end{array}\right)=\left(\begin{array}{cc}
\cos \theta_{S} & -\sin \theta_{S} \\
\sin \theta_{S} & \cos \theta_{S}
\end{array}\right)\left(\begin{array}{l}
h^{\prime} \\
s^{\prime}
\end{array}\right)
$$

where for the scalar mixing angle $\theta_{S} \in\left(-\frac{\pi}{4}, \frac{\pi}{4}\right)$ we find

$$
\tan \left(2 \theta_{S}\right)=-\frac{\lambda v w}{\lambda_{\phi} v^{2}-\lambda_{\chi} w^{2}}
$$

The squares of the masses of the mass eigenstates $h$ and $s$ $\left(m_{h}\right.$ and $\left.m_{s}\right)$ are

$M_{h / H}^{2}=\lambda_{\phi} v^{2}+\lambda_{\chi} w^{2} \mp \sqrt{\left(\lambda_{\phi} v^{2}-\lambda_{\chi} w^{2}\right)^{2}+(\lambda v w)^{2}}$

where $M_{h} \leq M_{H}$ in out convention. At this point either $m_{h / s}=M_{h / H}$ or $m_{h / s}=M_{H / h}$.

As $M_{h}$ must be positive, the condition

$$
v^{2} w^{2}\left(4 \lambda_{\phi} \lambda_{\chi}-\lambda^{2}\right)>0
$$

has to be fulfilled. If both VEVs are greater than zero-as needed for two nonvanishing scalar masses-, then this condition reduces to the positivity constraint (7), but with different meaning. Equation (7) is required to ensure that the potential be bounded from below if $\lambda<0$, which has to be fulfilled at any scale. For $\lambda>0$, the potential is bounded from below even without requiring the constraint (7). The inequality in (17) ensures $M_{h}>0$, which has to be fulfilled as long as $v w>0$ independently of the sign of $\lambda$.

The VEV of the BEH field and the mass of the Higgs boson (at the scale equal to mass of the t-quark) are known experimentally, $v\left(m_{\mathrm{t}}\right) \simeq 262 \mathrm{GeV}$ and $m_{H}\left(m_{\mathrm{t}}\right) \simeq 131.55 \mathrm{GeV}$ [8]. Introducing the abbreviation $\lambda_{\mathrm{SM}}=\frac{1}{2} m_{H}^{2} / v^{2}$, we have $\lambda_{\mathrm{SM}}\left(m_{\mathrm{t}}\right) \simeq 0.126$ and we can distinguish two cases at the weak scale: (i) $\lambda_{\phi}\left(m_{\mathrm{t}}\right)>$ $\lambda_{\text {SM }}\left(m_{\mathrm{t}}\right)$ and (ii) $\lambda_{\mathrm{SM}}\left(m_{\mathrm{t}}\right)>\lambda_{\phi}\left(m_{\mathrm{t}}\right)$. Then we can relate the new VEV $w$ to the BEH VEV $v$ and the four couplings $\lambda_{\mathrm{SM}}, \lambda_{\phi}, \lambda_{\chi}, \lambda$ using Eq. (16) as

$$
\begin{gathered}
w\left(m_{\mathrm{t}}\right)^{2}\left(4\left(\lambda_{\phi}\left(m_{\mathrm{t}}\right)-\lambda_{\mathrm{SM}}\left(m_{\mathrm{t}}\right)\right) \lambda_{\chi}\left(m_{\mathrm{t}}\right)-\lambda\left(m_{\mathrm{t}}\right)^{2}\right) \\
=4 v\left(m_{\mathrm{t}}\right)^{2} \lambda_{\mathrm{SM}}\left(m_{\mathrm{t}}\right)\left(\lambda_{\phi}\left(m_{\mathrm{t}}\right)-\lambda_{\mathrm{SM}}\left(m_{\mathrm{t}}\right)\right) .
\end{gathered}
$$

Using Eq. (18), it is convenient to consider $w$ as a dependent parameter and scan the parameter space of the remaining three quartic couplings as done below. We are not interested in the case of $\lambda_{\phi}\left(m_{\mathrm{t}}\right)=\lambda_{\mathrm{SM}}\left(m_{\mathrm{t}}\right)$ because that prevents the model from interpreting neutrino masses [24].

In case (i) when $\lambda_{\phi}\left(m_{\mathrm{t}}\right)>\lambda_{\mathrm{SM}}\left(m_{\mathrm{t}}\right)$, then $M_{H}>m_{H}$, so only $h$ can be the Higgs particle and $M_{h}=m_{H}$, while 


$$
M_{H}=m_{H} \sqrt{\frac{\lambda_{\phi}-\lambda_{\mathrm{SM}}}{\lambda_{\mathrm{SM}}}} \sqrt{\frac{4 \lambda_{\phi} \lambda_{\chi}-\lambda^{2}}{4\left(\lambda_{\phi}-\lambda_{\mathrm{SM}}\right) \lambda_{\chi}-\lambda^{2}}} .
$$

The positivity of $M_{H}^{2}$, in addition to the constraint in (17), also requires that

$$
4\left(\lambda_{\phi}-\lambda_{\mathrm{SM}}\right) \lambda_{\chi}-\lambda^{2}>0 \quad \text { or } \quad \lambda_{\phi}>\lambda_{\mathrm{SM}}+\frac{\lambda^{2}}{4 \lambda_{\chi}} .
$$

In case (ii), $m_{H}^{2}>2 \lambda_{\phi} v^{2}>M_{h}^{2}$, so only $H$ can be the Higgs particle and we can express the masses of the scalars as in Eq. (19), with $h$ and $H$ interchanged, or explicitly

$$
M_{h}=m_{H} \sqrt{\frac{\lambda_{\mathrm{SM}}-\lambda_{\phi}}{\lambda_{\mathrm{SM}}}} \sqrt{\frac{4 \lambda_{\phi} \lambda_{\chi}-\lambda^{2}}{\lambda^{2}+4\left(\lambda_{\mathrm{SM}}-\lambda_{\phi}\right) \lambda_{\chi}}}
$$

and $M_{H}=m_{H}$, which does not require any further constraint to (17).

In principle, it may happen that one of the VEVs vanishes at some critical scale $t_{c}$. In that case, for $t>t_{c}$ the only scalar particle is the Higgs boson. Thus, beyond $t_{c}$ we do not need to assume the validity of the extra constraints beyond the requirements of stability and the new scalar sector affects only the RG equations.

\section{B. Neutrino Yukawa sector}

Neutrino oscillation experiments prove that neutrinos have masses, which in a usual gauge field theoretical description necessitates the assumption that right-handed neutrinos exist. The existence of the new scalar allows for gauge invariant Majorana-type Yukawa terms of dimension four operators for the neutrinos

$$
\mathcal{L}_{\mathrm{Y}}^{\nu}=-\frac{1}{2} \sum_{i, j} \overline{\nu_{i, \mathrm{R}}^{c}}\left(c_{\mathrm{R}}\right)_{i j} \nu_{j, \mathrm{R}} \chi+\text { h.c. }
$$

provided the superscript $c$ denotes the charge conjugate of the field. The Yukawa coupling matrix $\left(c_{\mathrm{R}}\right)_{i j}$ is a real symmetric matrix whose values are not constrained. There are other gauge invariant Yukawa terms involving the left-handed neutrinos (see Ref. [24] where all possible terms are taken into account for neutrino mass generation), but those must contain small Yukawa couplings, otherwise the left-handed neutrino masses would violate experimental constraints. In our analysis below we assume that at least one element of the diagonal matrix $O c_{\mathrm{R}} O^{T}$, with $O$ being a suitable orthogonal matrix, can take any value in the range $(0,2)$. We denote this element by $c_{\nu}$ below.

\section{RENORMALIZATION GROUP EQUATIONS}

In order to study the values of the couplings at any energy scale we use the RG equations,

$$
\frac{\mathrm{d} g}{\mathrm{~d} t}=a \beta(g)
$$

where the factor $a=\ln 10$ ensures that the RG-time $t=$ $\ln (\mu / \mathrm{GeV})$ represents the energy scale $\mu=10^{t} \mathrm{GeV}$ rather than $\mu=e^{t} \mathrm{GeV}$ and the variable $g$ is a generic notation for any of the five gauge couplings, the four most relevant Yukawa couplings $c_{\mathrm{t}}, c_{\mathrm{b}}, c_{\tau}$, and $c_{\nu}$, the two quadratic and three quartic scalar couplings (14 equations in total). In order to solve this coupled system of differential equations, we need to specify the $\beta$-functions and the initial conditions for the couplings.

At one-loop in perturbation theory, the $\beta$-function of a dimensionless coupling $g$ is computed from the formula

$$
\beta_{0}(g)=M \frac{\partial}{\partial M}\left(-\delta_{g}+\frac{1}{2} g \sum_{i} \delta_{Z, i}\right)
$$

where $\delta_{g}$ is the one-loop counterterm for a given vertex, which is proportional to $g$, while $\delta_{Z, i}$ are the wave function renormalization counterterms for all the $i$ legs of the given vertex. The one-loop $\beta$-functions are independent of the renormalization scheme and so is the one-loop equation Eq. (24) (see for instance Chapter 12 of Ref. [26]). We computed those in perturbation theory at one-loop order for the complete model of Ref. [24], and can be found in Ref. [27].

In order to obtain the running of the scalar couplings, we need the $\beta$-functions of the scalar sector. According to our assumption on the smallness of the new gauge couplings, we can set $g_{Z}=0$. We also neglect the Yukawa couplings of all charged leptons as well as the quarks, except that of the $\mathrm{t}$-quark. With these assumptions the $\beta$-functions $\beta_{0}(g) \equiv b_{0}(g) /(4 \pi)^{2}$ of the gauge and Yukawa couplings simplify to their forms in the standard model, while those in the scalar sector become

$$
\begin{aligned}
b_{0}\left(\mu_{\phi}^{2}\right) & =\mu_{\phi}^{2}\left(12 \lambda_{\phi}+2 \frac{\mu_{\chi}^{2}}{\mu_{\phi}^{2}} \lambda+6 c_{\mathrm{t}}^{2}-\frac{3}{2} g_{Y}^{2}-\frac{9}{2} g_{\mathrm{L}}^{2}\right), \\
b_{0}\left(\mu_{\chi}^{2}\right) & =\mu_{\chi}^{2}\left(8 \lambda_{\chi}+4 \frac{\mu_{\phi}^{2}}{\mu_{\chi}^{2}} \lambda+\frac{1}{2} c_{\nu}^{2}\right) \quad \text { with Dirac } \\
& =\mu_{\chi}^{2}\left(8 \lambda_{\chi}+4 \frac{\mu_{\phi}^{2}}{\mu_{\chi}^{2}} \lambda+c_{\nu}^{2}\right) \quad \text { with Majorana }
\end{aligned}
$$

neutrino for the quadratic, and 


$$
\begin{aligned}
b_{0}\left(\lambda_{\phi}\right) & =24 \lambda_{\phi}^{2}+\lambda^{2}-6 c_{\mathrm{t}}^{4}+\frac{3}{8} g_{Y}^{4}+\frac{9}{8} g_{\mathrm{L}}^{4}+\frac{3}{4} g_{Y}^{2} g_{\mathrm{L}}^{2}-\lambda_{\phi}\left(9 g_{\mathrm{L}}^{2}+3 g_{Y}^{2}\right)+12 \lambda_{\phi} c_{\mathrm{t}}^{2} \\
b_{0}\left(\lambda_{\chi}\right) & =20 \lambda_{\chi}^{2}+2 \lambda^{2}-\frac{1}{8} c_{\nu}^{4}+\lambda_{\chi} c_{\nu}^{2} \quad \text { with Dirac neutrino, } \\
& =20 \lambda_{\chi}^{2}+2 \lambda^{2}-\frac{1}{2} c_{\nu}^{4}+2 \lambda_{\chi} c_{\nu}^{2} \quad \text { with Majorana neutrino, } \\
b_{0}(\lambda) & =12 \lambda \lambda_{\phi}+8 \lambda \lambda_{\chi}+4 \lambda^{2}+\lambda\left(\frac{1}{4} c_{\nu}^{2}+6 c_{\mathrm{t}}^{2}-\frac{9}{2} g_{\mathrm{L}}^{2}-\frac{3}{2} g_{Y}^{2}\right) \quad \text { with Dirac neutrino, } \\
& =12 \lambda \lambda_{\phi}+8 \lambda \lambda_{\chi}+4 \lambda^{2}+\lambda\left(c_{\nu}^{2}+6 c_{\mathrm{t}}^{2}-\frac{9}{2} g_{\mathrm{L}}^{2}-\frac{3}{2} g_{Y}^{2}\right) \quad \text { with Majorana neutrino }
\end{aligned}
$$

for the quartic couplings where $g_{\mathrm{L}}$ and $g_{Y}$ are the lefthanded and hypercharge couplings. We solve this system of simplified equations numerically for both types of neutrinos. Of course, for $c_{\nu}=0$ the difference between the equations for Dirac and Majorana neutrinos disappears. For $c_{\nu}>0$ the qualitative behavior of the running couplings is similar for the two types of neutrinos, but the larger coefficients in front of $c_{\nu}$ for the Majorana neutrino results in a stronger effect of the neutrino Yukawa coupling, and eventually more constrained parameter space.

We fix the initial conditions for the standard model couplings as done in the two-loop analysis of Ref. [8] (using the two-loop $\overline{\mathrm{MS}}$ scheme). Specifically, we set

$$
\begin{aligned}
g_{Y}\left(m_{\mathrm{t}}\right) & =\sqrt{\frac{3}{5}} \times 0.4626, \quad g_{\mathrm{L}}\left(m_{\mathrm{t}}\right)=0.648, \\
\lambda_{\mathrm{SM}}\left(m_{\mathrm{t}}\right) & =0.126, \quad v\left(m_{\mathrm{t}}\right)=262 \mathrm{GeV},
\end{aligned}
$$

and $c_{\mathrm{t}}\left(m_{\mathrm{t}}\right)=0.937$. Choosing some initial values of the quartic couplings $\lambda_{\phi}\left(m_{\mathrm{t}}\right), \lambda_{\chi}\left(m_{\mathrm{t}}\right)$ and $\lambda\left(m_{\mathrm{t}}\right)$, we obtain the couplings $\mu_{\phi}\left(m_{\mathrm{t}}\right)$ and $\mu_{\chi}\left(m_{\mathrm{t}}\right)$ according to Eq. (9), with

$$
w\left(m_{\mathrm{t}}\right)=2 v\left(m_{\mathrm{t}}\right) \sqrt{\frac{\lambda_{\mathrm{SM}}\left(m_{\mathrm{t}}\right)\left(\lambda_{\phi}\left(m_{\mathrm{t}}\right)-\lambda_{\mathrm{SM}}\left(m_{\mathrm{t}}\right)\right)}{\left(4\left(\lambda_{\phi}\left(m_{\mathrm{t}}\right)-\lambda_{\mathrm{SM}}\left(m_{\mathrm{t}}\right)\right) \lambda_{\chi}\left(m_{\mathrm{t}}\right)-\lambda\left(m_{\mathrm{t}}\right)^{2}\right)}},
$$

determined from Eq. (18).

\section{CONSTRAINTS FROM STABILITY AND PERTURBATIVITY}

In order to constrain the parameter space of the new couplings, spanned by $\lambda_{\phi}, \lambda_{\chi}, \lambda$ and $c_{\nu}$, we require the validity of the conditions of Table I, i.e., the stability of the vacuum up to the Planck scale $m_{\mathrm{P}}$. Such studies have already been presented for various hidden sector (usually singlet scalar) extensions of the standard model in Refs. [28-31]. In addition, we also check the validity of the constraints set by the positivity requirement on the scalar masses [Eq. (20) for case (i) and Eq. (17) for case (ii)], from the initial conditions up to $m_{\mathrm{P}}$, but as long as $w>0$. A similar analysis was presented in Ref. [32], but with $Z_{2}$ symmetry assumed on the new gauge sector. Our analysis is based on the simplest, but complete (in the sense of renormalizable quantum field theory) extension of the standard model gauge group described in Ref. [24].

As seen in Eq. (26), the $\beta$-functions are independent of both $\mu_{\phi}$ and $\mu_{\chi}$, except of course their own $\beta$-functions, which decouple from the rest. Thus, in the parameter scan we focus on the four-dimensional parameter subspace of $c_{\nu}, \lambda_{\phi}, \lambda_{\chi}, \lambda$ by selecting slices at fixed values of $c_{\nu}$. In addition to the stability conditions, we also require that the couplings remain in the perturbative region that we defined by

$$
\lambda_{\phi}(t)<4 \pi, \quad \lambda_{\chi}(t)<4 \pi, \quad|\lambda(t)|<4 \pi .
$$

We have restricted the region of the new VEV to $w<1 \mathrm{TeV}$ because a large value of $w$ is likely to imply large kinetic mixing between the two $U(1)$ gauge fields [24], which is not supported by experiments (see, e.g., Ref. [33]). This restriction does not influence the allowed regions for the quartic couplings significantly.

Figures 1 and 2 display our results for the allowed regions for the initial conditions of $\lambda_{\phi}\left(m_{\mathrm{t}}\right), \lambda_{\chi}\left(m_{\mathrm{t}}\right)$ and $\lambda\left(m_{\mathrm{t}}\right)$ at three selected values of the Dirac neutrino Yukawa coupling as shaded areas where the stability of the vacuum and the constraints set by the positivity requirement on the scalar masses are respected. In order to ease the interpretation, we show projections of the allowed threedimensional region onto two-dimensional subspaces. We also show the running couplings up to the Planck scale at a point representing selected values of the initial conditions at the electroweak scale. Although the new VEV $w$ is not an independent parameter, we find interesting to present the projections also in the $w-g$ subspaces where $g$ denotes one of the quartic couplings (see Fig. 3). The foremost conclusion is that the parameter space is not empty, but only for case (i), i.e., when $\lambda_{\phi}\left(m_{\mathrm{t}}\right)>\lambda_{\mathrm{SM}}$. Thus the Higgs particle has the smaller scalar mass always. In fact, we find that the 

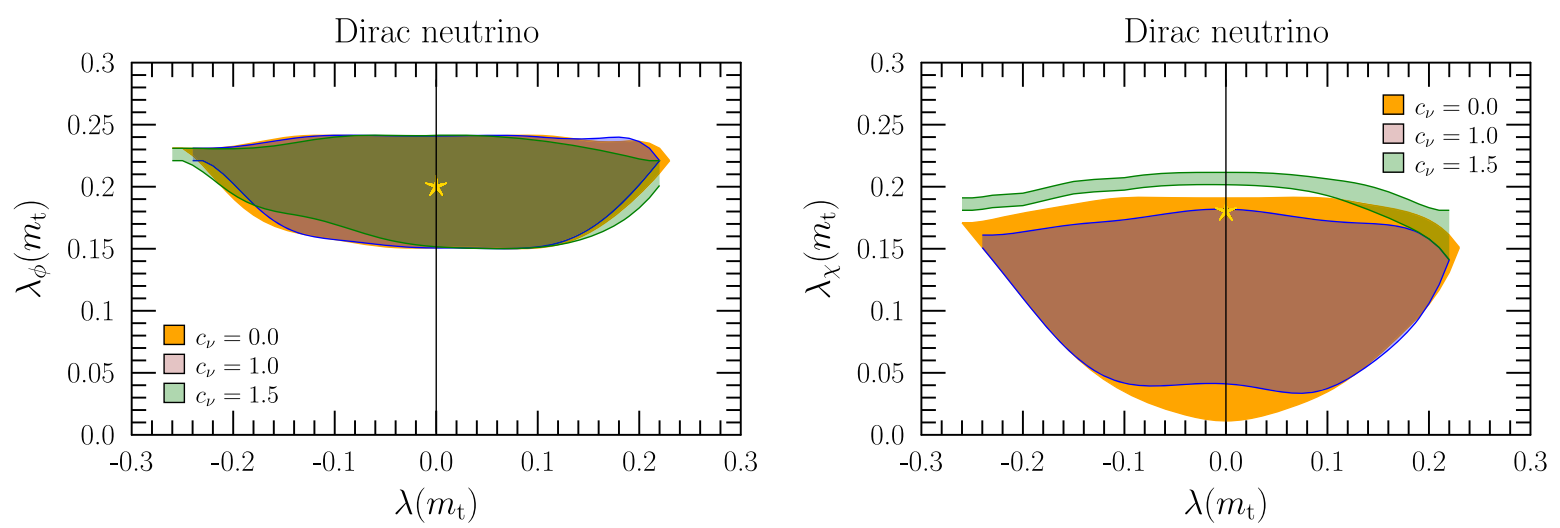

FIG. 1. Accepted initial conditions (as shaded areas) in the $\lambda_{\phi}\left(m_{\mathrm{t}}\right)-\lambda\left(m_{\mathrm{t}}\right)$ plane (left) and $\lambda_{\chi}\left(m_{\mathrm{t}}\right)-\lambda\left(m_{\mathrm{t}}\right)$ plane (right) for the stability of the vacuum and perturbativity preserved up to the Planck mass at different values of the Dirac neutrino Yukawa coupling $c_{\nu}$. The star marks the point in the parameter space for which the example of the running couplings up to the Planck scale is presented in Fig. 2.
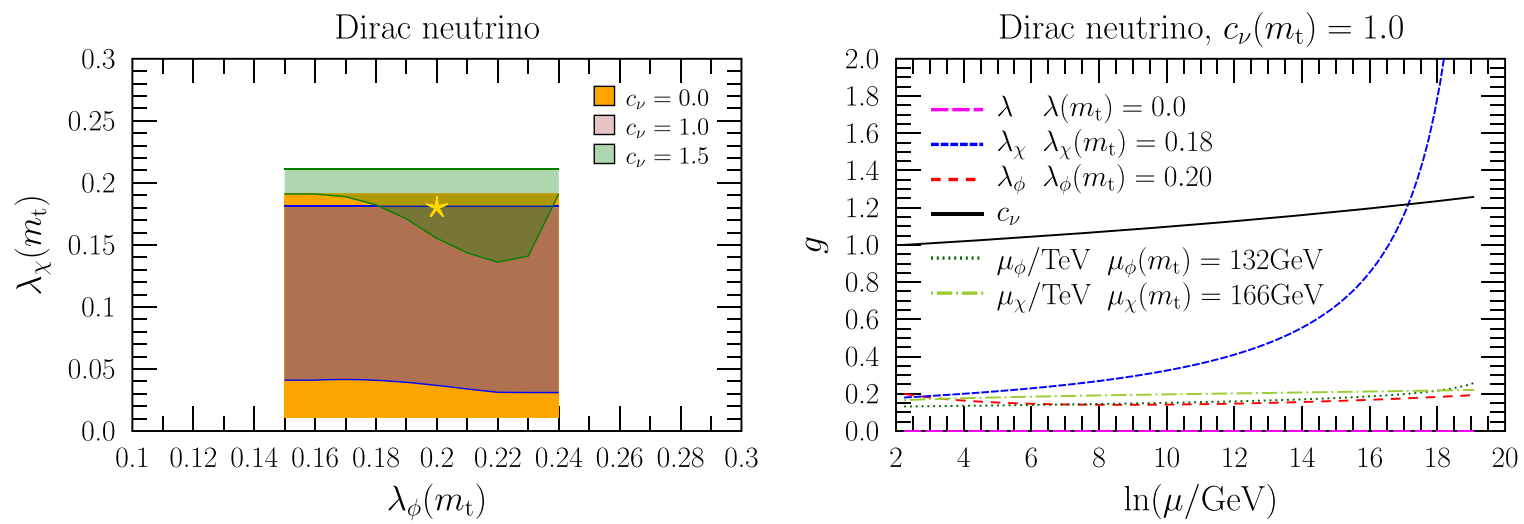

FIG. 2. Left: same as Fig. 1 in the $\lambda_{\phi}\left(m_{\mathrm{t}}\right)-\lambda_{\chi}\left(m_{\mathrm{t}}\right)$ plane. Right: the running of the couplings up to the Planck scale in a selected point of the parameter space.

allowed region for $\lambda_{\phi}\left(m_{\mathrm{t}}\right)$ is about [0.151, 0.241] (starting to decrease only for $c_{\nu}\left(m_{\mathrm{t}}\right)>1.5$, while $\min M_{H}\left(m_{\mathrm{t}}\right) \simeq$ $144 \mathrm{GeV}$. Clearly, the precise values may somewhat change in an analysis at the accuracy of higher loops. Even in the allowed region for $\lambda_{\phi}$, the parameter space for the other couplings is constrained significantly and decreases slowly with increasing Yukawa coupling of the right handed neutrino up to $c_{\nu} \simeq 1$. Above $c_{\nu} \simeq 1$ the parameter space vanishes swiftly. The maximal allowed regions for the parameters are presented for the selected values of $c_{\nu}$ in Table II. Thus we find that the stability of the vacuum requires $c_{\nu} \lesssim 1.65$ for Dirac neutrinos $\left(c_{\nu} \lesssim 1.15\right.$ for
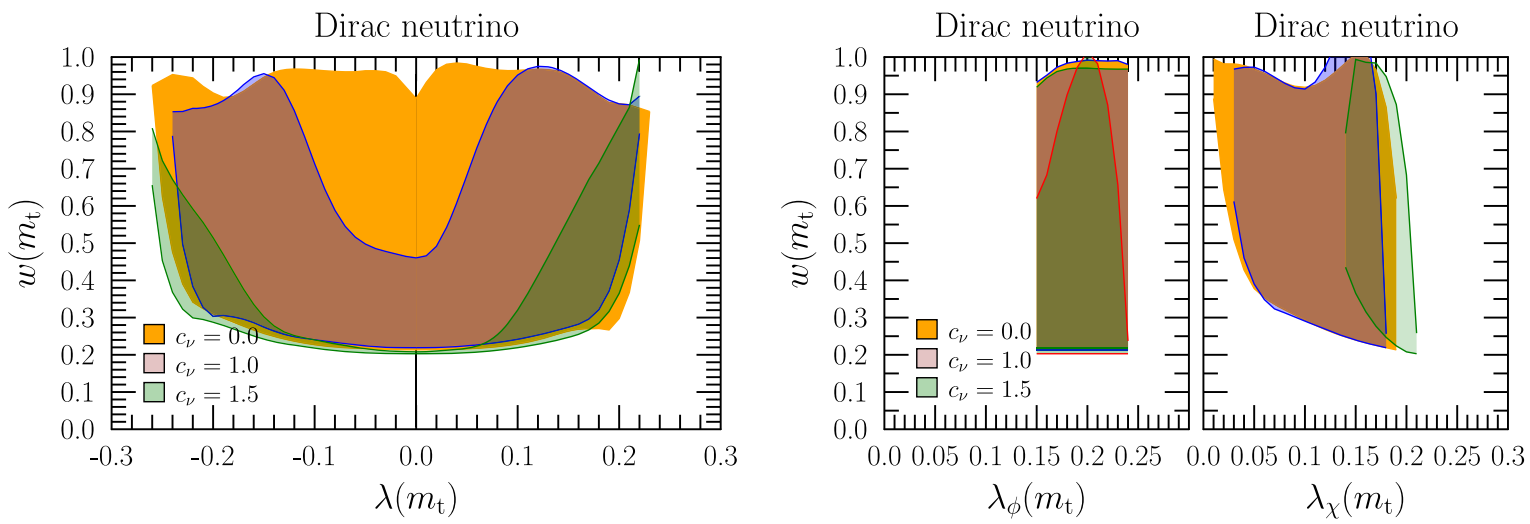

FIG. 3. Same as Fig. 1 in the $w\left(m_{\mathrm{t}}\right)-g\left(m_{\mathrm{t}}\right)$ planes. Left: $g=\lambda$, right: $g=\lambda_{\phi}$ and $\lambda_{\chi}$. 
TABLE II. Maximal allowed regions of the couplings required by stability of the vacuum and perturbativity of the couplings up to the Planck scale for selected values of the Yukawa coupling of the right-handed neutrino $c_{\nu}\left(m_{\mathrm{t}}\right)$ and $w<1$ TeV set explicitly.

\begin{tabular}{lcccccc}
\hline \hline$c_{\nu}$ & $\lambda(t)$ & $\lambda_{\chi}(t)$ & $\left(\mu_{\phi}(t) / \mathrm{GeV}\right)^{2}$ & $\left(\mu_{\chi}^{2}(t) / \mathrm{GeV}\right)^{2}$ & $w(t) / \mathrm{GeV}$ & $M_{H}(t) / \mathrm{GeV}$ \\
\hline 0.0 & {$[-0.26,0.23]$} & {$[0.011,0.191]$} & {$\left[-436^{2}, 496^{2}\right]$} & {$\left[112^{2}, 580^{2}\right]$} & {$[213,1000]$} & {$[144,558]$} \\
1.0 & {$[-0.24,0.22]$} & {$[0.031,0.181]$} & {$\left[-436^{2}, 436^{2}\right]$} & {$\left[114^{2}, 548^{2}\right]$} & {$[220,1000]$} & {$[144,557]$} \\
1.5 & {$[-0.26,0.22]$} & {$[0.141,0.211]$} & {$\left[-374^{2}, 496^{2}\right]$} & {$\left[111^{2}, 603^{2}\right]$} & {$[203,994]$} & {$[144,598]$} \\
\hline \hline
\end{tabular}

Majorana neutrinos). It is also interesting to remark that the allowed regions are also very sensitive to the value of the Yukawa coupling of the t quark. For instance, at $c_{\mathrm{t}}\left(m_{\mathrm{t}}\right) \simeq 1.1$ the allowed parameter space vanishes completely.

\section{COSMOLOGICAL INFLATION}

We now explore the cosmic inflation of the two-field model with potential energy defined in Eq. (4). We consider slow-roll inflation when the potential energy has a large, almost flat area for small field values and a global minimum at large values of the VEVs. Such a potential energy allows for slow roll of the fields from small values toward the global minimum, resulting in cosmic inflation. The required form of the potential energy function appears naturally at some high energy scale, for certain values of the scalar couplings at the mass of the t-quark $m_{\mathrm{t}}$. As Eq. (8) shows, the VEVs are inversely proportional to $\sqrt{\operatorname{det} C}$. Fig. 4 shows the running of $\operatorname{det} C$ together with that of the couplings from initial values at $m_{\mathrm{t}}$ chosen from the stability region. We see a narrow wedge-like an inverse resonance-where $\operatorname{det} C$ becomes very small, implying VEVs at around field values of $10^{5} \mathrm{GeV}$. The figure shows an example with vanishing Yukawa coupling $c_{\nu}$ of the righthanded neutrino, but below we show that the value of $c_{\nu}$ influences only the size of the parameter space of the scalar couplings where this phenomenon leads to such potential energy function that can support cosmic inflation in accordance with current values of relevant observables.

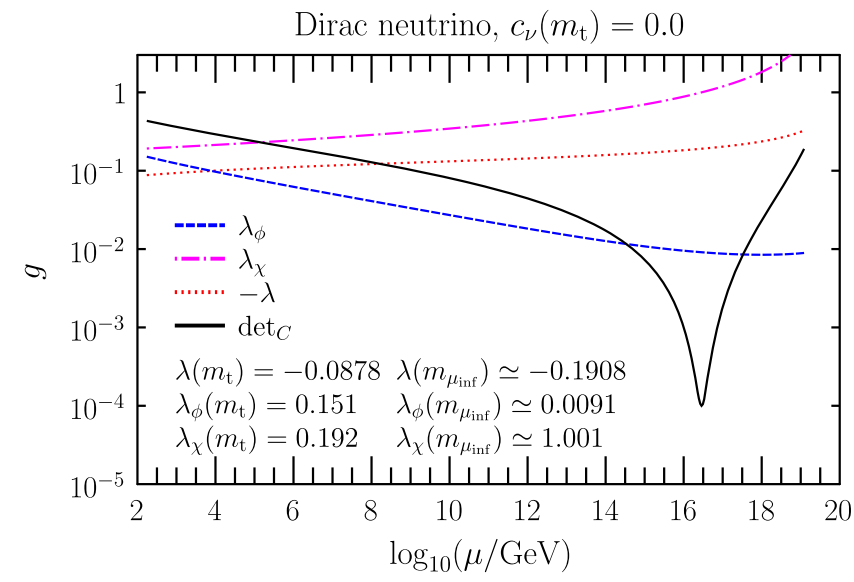

FIG. 4. Running of the scalar couplings and of $\operatorname{det} C . g$ means any of the couplings.
The single-field inflationary models predict purely curvature perturbations, resulting from energy density fluctuations. Having multiple fields allows for multiple types of fluctuations, hence several observable quantities, such as the tilts corresponding to curvature, isocurvature (emerging due to fluctuations in the relative number density of particles) and a correlation angle $\Delta$ [34].

Following Ref. [35], we introduce a local rotation of $(\phi, \chi)$ into $(\sigma, s)$, with $\sigma$ and $s$ referring to the adiabatic and entropy field. The adiabatic field is the path length along the classical trajectory, while $s$ remains a constant. Using the power spectra $\mathcal{P}_{R}(k)$ and $\mathcal{P}_{S}(k)$ of these comoving curvature and isocurvature fluctuations [36], as well as the spectrum of the cross-correlation $\mathcal{C}_{R S}(k)$ of the two fields, the correlation angle is defined as the dimensionless ratio

$$
\cos \Delta=\frac{\mathcal{C}_{R S}(k)}{\mathcal{P}_{R}^{1 / 2}(k) \mathcal{P}_{S}^{1 / 2}(k)} .
$$

As the fluctuations can communicate in super-horizon scales, in order to acquire the observable primordial fluctuations one has to evolve in time the curvature $R_{*}$ and isocurvature fluctuations $S_{*}$ calculated at horizon exit. This is achieved by applying the transfer matrix [34]:

$$
\left(\begin{array}{c}
R \\
S
\end{array}\right)=\left(\begin{array}{cc}
1 & T_{R S} \\
0 & T_{S S}
\end{array}\right)\left(\begin{array}{c}
R_{*} \\
S_{*}
\end{array}\right)
$$

The $T_{R S}$ and $T_{S S}$ transfer coefficients are related to the correlation angle. At the first order in the slow-roll expansion, we have

$$
\cos \Delta \simeq \frac{T_{R S}}{\sqrt{1+T_{R S}^{2}}} \quad \text { and } \quad \sin \Delta \simeq \frac{1}{\sqrt{1+T_{R S}^{2}}}
$$

Also, at the leading order of the slow-roll expansion the curvature power spectrum is

$$
\mathcal{P}_{R}=\mathcal{P}_{R^{*}}\left(1+T_{R S}^{2}\right),
$$

so $\mathcal{P}_{R^{*}}=\mathcal{P}_{R} \sin ^{2} \Delta$. As a result, a factor of $\sin ^{2} \Delta$ appears in the tensor-to-scalar ratio, as compared to the single-field case: $r=16 \epsilon(\sin \Delta)^{2}$.

The number of slow-roll parameters also increase. In the single-field case, in addition to the parameter $\epsilon=\frac{3}{2}\left(\frac{p}{\rho}+1\right)$, 

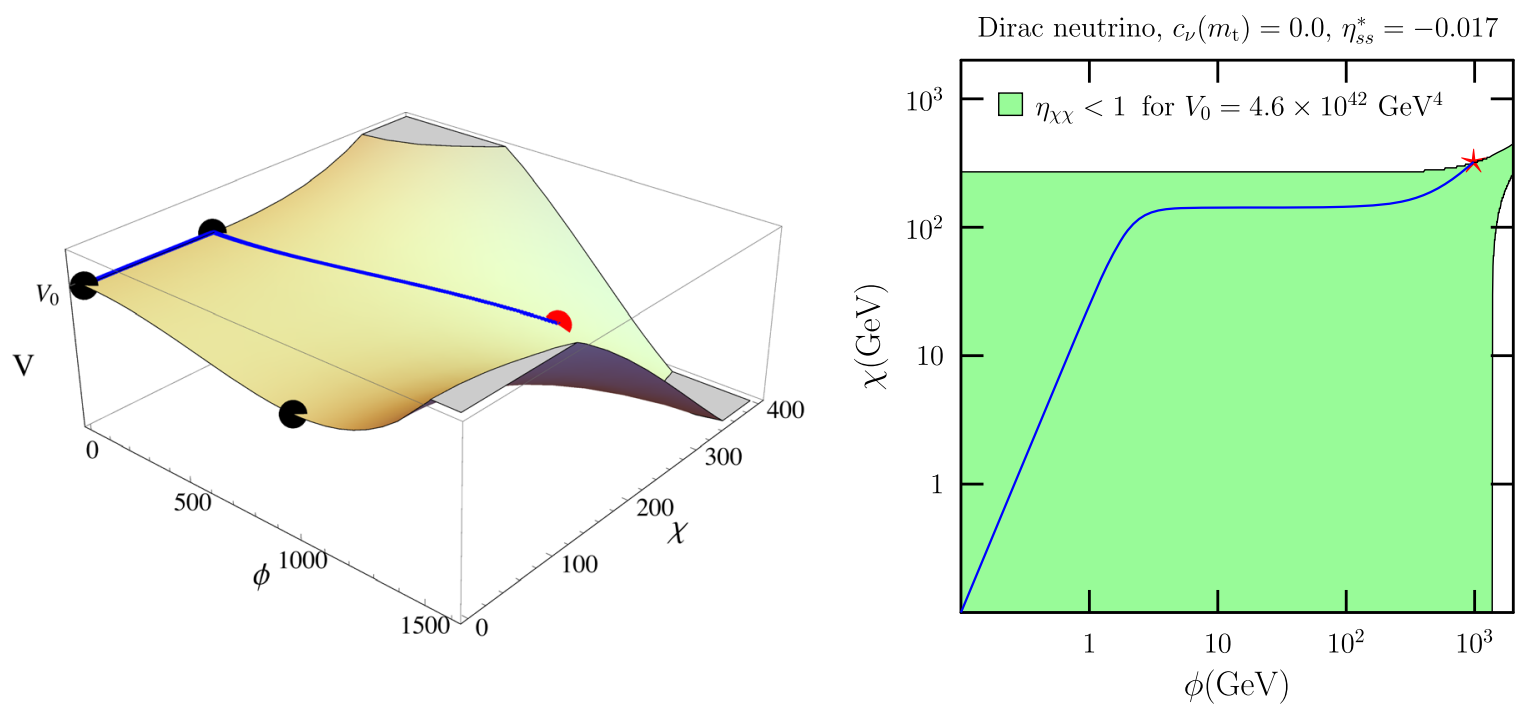

FIG. 5. Left: A possible trajectory of the rolling of the scalar fields. The black dots denote the extrema of the potential. Right: Projection of the trajectory onto the $\phi-\chi$ plane. The red star denotes the end of the inflation on the trajectory, marked with a red dot on the three-dimensional picture.

describing the deviation from the equation of state of the de Sitter space-time $(\rho=-p$, with $\rho$ denoting the energy density and $p$ is the pressure), there is only one other slowroll parameter $\eta$, which essentially measures the acceleration of the fields. In our example we have three $\eta$ parameters that can be expressed approximately from the potential as

$$
\eta_{i j} \simeq m_{\mathrm{P}}{ }^{2} \frac{\partial_{i j} V}{V}, \quad i j=\phi \phi, \chi \chi, \phi \chi, s s, \sigma \sigma, s \sigma
$$

(note that $\eta_{\phi \phi}+\eta_{\chi \chi}=\eta_{\sigma \sigma}+\eta_{s s}$ ), while

$$
\epsilon \simeq \frac{1}{2} m_{\mathrm{P}}^{2}\left(\frac{\partial_{\sigma} V}{V}\right)^{2} .
$$

In principle, inflation is possible only until both $\epsilon$ and $\eta_{i j}$ are small, resulting in the slow roll.

To set the exact conditions of slow roll, we numerically solved the equations of motion, namely

$$
\begin{aligned}
& \ddot{\phi}+3 H \dot{\phi}+\frac{\partial V}{\partial \phi}=0, \\
& \ddot{\chi}+3 H \dot{\chi}+\frac{\partial V}{\partial \chi}=0,
\end{aligned}
$$

with the integration variable transformed to the number of e-folds $N$, and terminated the process, when any of the slow-roll parameters reached unity. We set the starting point of the trajectory at vanishing field values. For the parameter values of the potential energy we used values allowed by the perturbativity and stability conditions mentioned in Sec. II, namely $|\lambda|, \lambda_{i} \sim \mathrm{O}\left(10^{-1}-10^{-2}\right)$ and $\mu_{i}^{2} / \mathrm{GeV}^{2} \sim \mathrm{O}\left(1-3 \times 10^{4}\right)$. For such values we have found that the $\eta_{i j}$ parameters increase much faster than $\epsilon$, reaching 1, while $\epsilon$ remaining small, about $\mathrm{O}\left(10^{-30}\right)$. Hence, we set the end of inflation by the condition $\eta_{i j}=1$. In practice the parameter $\eta_{\chi \chi}$ increases the fastest. We show an example of such a trajectory in Fig. 5. This trajectory induces $N>200$ e-folds. The value of $\eta_{s s}^{*}$ refers to the value of $\eta_{s s}$ at 60 e-folds before the end of inflation.

The smallness of $\epsilon$ in our model contradicts to the swampland conjecture that states that it has to be $\mathrm{O}(1)$ during inflation [37]. If the conjecture finds a proof, then our model will be excluded, but at present it is not yet the case. Strictly speaking, this conjecture, if it were true, would forbid the slow-roll models of inflation, which stimulated a lot of activity aiming at refining the conjecture (see, e.g., [38,39]). In this context, we have not studied if our phenomenological model remains a consistent theory at the Planck scale, and thus it has similar status as many other studies of inflationary models. The model of slow-roll inflation we propose here is based on a scalar potential that is not connected to an exact de Sitter vacuum, but have an equation of state that resembles that of the de Sitter vacuum, with $\epsilon=0$ meaning exact coincidence. The very small value of $\epsilon$ implies exponential inflation, whose end is marked by the increasing acceleration of the new scalar field $\chi$, giving a distinct role (or necessity) for $\chi$ in the particle physics model (in addition to stabilizing the vacuum up to the Planck scale).

The observables are constructed from the slow-roll parameters taken 50-60 e-folds before the end of inflation. This corresponds to an even smaller $\epsilon$, which reduces the tensor-to-scalar ratio, $r=16 \epsilon(\sin \Delta)^{2}$ to essentially zero. Such a small $r$ is not excluded by current cosmological 
measurements. The smallness of $r$ however is in conflict with the traditional cosmological normalization

$$
V_{0} \simeq r\left(1.6 \times 10^{16} \mathrm{GeV}\right)^{4} .
$$

This conflict may be resolved by assuming that the adiabatic and entropy fluctuations were maximally correlated at 50-60 e-folds before the end of inflation, implying $\cos \Delta=1$, and hence predicting zero for the tensor-toscalar ratio. The case of $\Delta=0$, i.e., maximally correlated fluctuations are referred to as the curvaton scenario. In this case, the various tilts coincide.
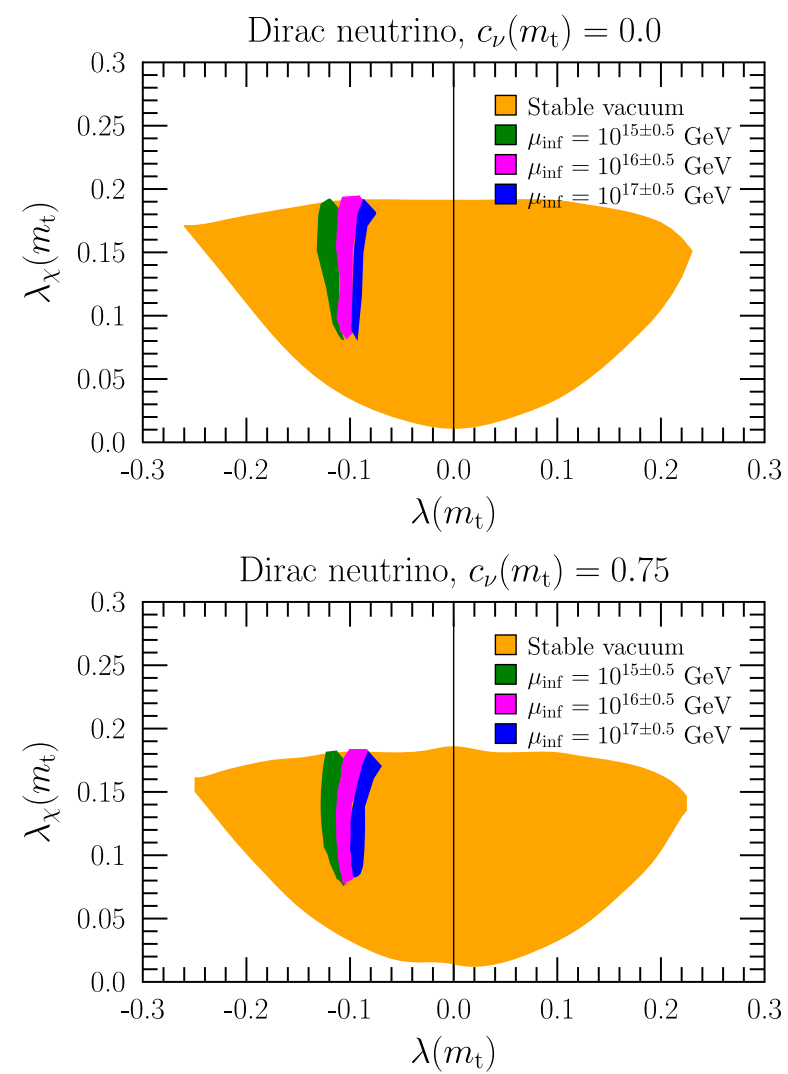

Dirac neutrino, $c_{\nu}\left(m_{\mathrm{t}}\right)=1.0$

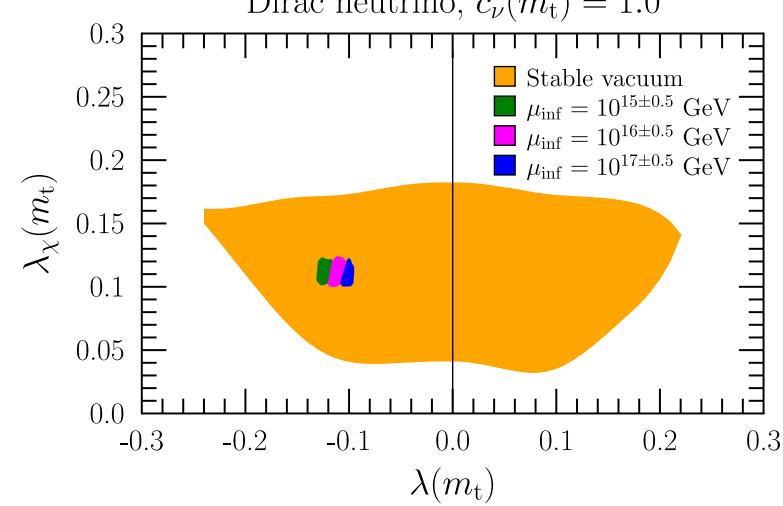

In the curvaton scenario we have to find a new condition to set the scale of inflation $\left(V_{0}\right)^{1 / 4}$. Neglecting $\epsilon$ we have:

$$
n_{s}-1=2 \eta_{s s}
$$

Considering $\eta_{s s}$ as a function of $V_{0}$ (see Eq. (34) with $V_{0}$ in $V$ in the denominator), we normalize it to produce the scalar tilt in agreement with the most recent data, $n_{s} \simeq 0.966$, yielding $V_{0} \simeq 5 \times 10^{42} \mathrm{GeV}^{4}$, which corresponds to the a scale of inflation $V_{0}^{1 / 4} \simeq 5 \times 10^{10} \mathrm{GeV}$.

Having fixed the value of $V_{0}$, we propose the following inflationary scenario. The scalar potential energy is given
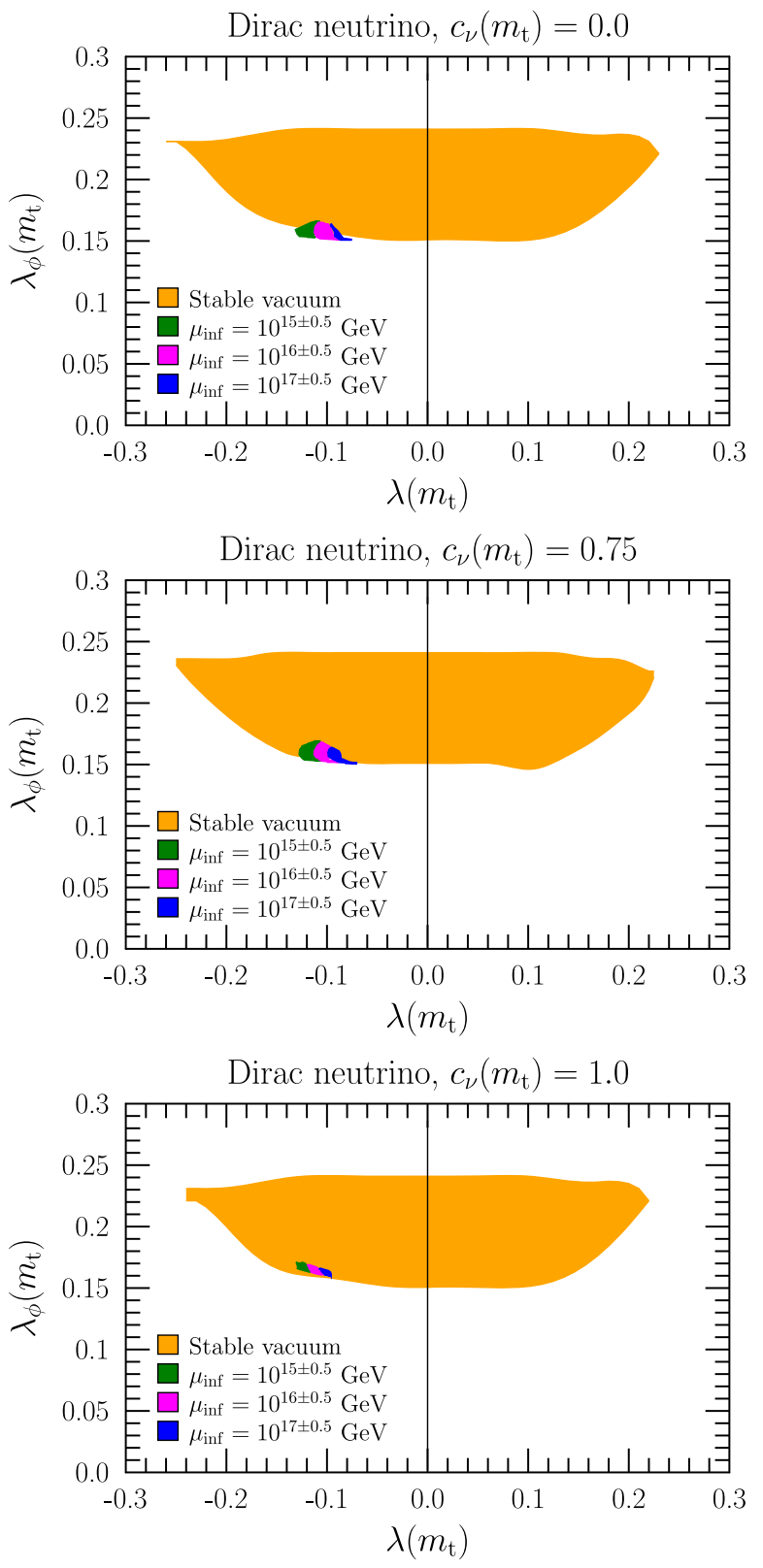

FIG. 6. Projections of the allowed regions of the parameter space of scalar couplings onto the $\lambda-\lambda_{\chi}$ plane (left) and $\lambda-\lambda_{\phi}$ plane (right) at three different ranges of the scale of inflation and at three selected values of the $c_{\nu}$ Yukawa coupling. 
by Eq. (4). After the big bang the characteristic energy scale of particle interactions is near the Planck scale, hence the scalar fields are fluctuating around zero. As the universe expands, the characteristic energy scale decreases and the scalar couplings run according to their renormalization group equations, exhibiting the wedge for $\operatorname{det} C$ at an energy scale $\mu_{\text {inf }}$ (around $10^{16} \mathrm{GeV}$ ) that we call the characteristic energy scale of inflation [40]. At this scale the global minimum of the potential energy function increases to about $10^{5} \mathrm{GeV}$ and the fields start to roll slowly toward this minimum, resulting in cosmic inflation. This accelerated expansion continues until the acceleration (second time derivative of the fields) remain negligible in the equation of the motion, determined by $\max \eta_{i j}=1$. After this the universe starts its Hubble expansion, decreasing the characteristic energy scale, and the global minimum of the scalar potential quickly returns to small field values as observed today, preventing any further inflationary periods.

\section{PREDICTING THE SCALAR COUPLINGS}

The cosmological inflation as described in the previous section occurs only in a restricted region of the parameter space of the scalar couplings, which we define at the electroweak scale $\left(m_{\mathrm{t}}\right)$.

The wedge in the running of $\operatorname{det} C$ appears only for $\lambda\left(m_{\mathrm{t}}\right)<0$. We have scanned this side of the parameter space by selecting fixed initial values for the scalar couplings $\lambda_{\phi}\left(m_{\mathrm{t}}\right), \lambda_{\chi}\left(m_{\mathrm{t}}\right)$, and scanning the allowed initial values of the third one to find those points where the wedge in the running of $\operatorname{det} C$ appears with a minimum $\lesssim \mathrm{O}\left(10^{-4}\right)$. During this scanning, we need to search only for those points where $w\left(m_{\mathrm{t}}\right)$-given by Eq. (28), with $\lambda_{\mathrm{SM}}\left(m_{\mathrm{t}}\right)=\frac{1}{2} m_{H}\left(m_{\mathrm{t}}\right)^{2} / v\left(m_{\mathrm{t}}\right)^{2} \simeq 0.126$ - and the masses of the scalars given by Eq. (16) remain positive. We find that the parameter space is constrained to a shell on the surface of the region allowed by the conditions of stability and perturbativity of $V$. The width of the shell is affected by the allowed depth of the minimum of $\operatorname{det} C$ : the smaller $\operatorname{det} C$, the thinner the shell. Furthermore, we have also found that the minimum value of the location of the wedge $\mu_{\text {inf }}$ is around $10^{14} \mathrm{GeV}$, depending slightly on $c_{\nu}\left(m_{\mathrm{t}}\right)$.
In Fig. 6 we present the results of such scan of the parameter space. These plots show different planar projections of the three dimensional parameter space, spanned by $\lambda_{\phi}\left(m_{\mathrm{t}}\right), \lambda_{\chi}\left(m_{\mathrm{t}}\right)$, and $\lambda\left(m_{\mathrm{t}}\right)$. The shape and size of the supported regions is affected by the choice of $c_{\nu}\left(m_{\mathrm{t}}\right)$, as seen in the titles of the figures. We find that the parameter space of the scalar couplings is not empty, but constrained strongly if we assume that cosmic inflation took place as described above. This assumption constrains the smallest value of $w\left(m_{\mathrm{t}}\right)$ to around $265 \mathrm{GeV}$.

\section{CONCLUSIONS}

We proposed a particle physics model of cosmic inflation. It requires at least two scalar fields. We explored the parameter space where the scalar potential remains bounded from below up to the Planck scale. We found that in a small region of the parameter space of the scalar couplings allowed by the requirement of stability, the determinant of the scalar quartic coupling matrix becomes very small at an energy scale around $10^{16} \mathrm{GeV}$. As a result the field values at the global minimum of the scalar potential increase significantly, allowing for an accelerated expansion of the universe by a slow-roll model of inflation. We assume the curvaton scenario of inflation, i.e., maximally correlated adiabatic and entropy fluctuations at 50-60 e-folds before the end of inflation, which implies vanishing tensor-to-scalar ratio. To set the scale of inflation (the normalization of the potential at vanishing field values), we required that the model reproduces the measured value of the scalar tilt. The inflation stops when the parameter that measures the acceleration of the fields starts to increase quickly. After this the position of the global minimum of the potential decreases preventing the appearance of another period of inflation. The model is consistent with observations as long as the tensor-to-scalar ratio is measured to be very small, $r \ll 1$.

\section{ACKNOWLEDGMENTS}

This work was supported by Grant No. K 125105 of the National Research, Development and Innovation Fund in Hungary.
[1] S. Weinberg, Phys. Rev. Lett. 19, 1264 (1967).

[2] S. Schael et al. (ALEPH, DELPHI, L3, OPAL, SLD Collaborations, LEP Electroweak Working Group, SLD Electroweak Group, SLD Heavy Flavour Group), Phys. Rep. 427, 257 (2006).

[3] Atlas experiment-Public results, https://twiki.cern.ch/twiki/ bin/view/AtlasPublic/StandardModelPublicResults.
[4] Summaries of cms cross section measurements, https://twiki.cern.ch/twiki/bin/view/CMSPublic/ PhysicsResultsCombined.

[5] G. Aad et al. (ATLAS Collaboration), Phys. Rev. D 90, 052004 (2014).

[6] V. Khachatryan et al. (CMS Collaboration), Eur. Phys. J. C 75, 212 (2015). 
[7] G. Degrassi, S. Di Vita, J. Elias-Miro, J. R. Espinosa, G. F. Giudice, G. Isidori, and A. Strumia, J. High Energy Phys. 08 (2012) 098.

[8] D. Buttazzo, G. Degrassi, P. P. Giardino, G. F. Giudice, F. Sala, A. Salvio, and A. Strumia, J. High Energy Phys. 12 (2013) 089.

[9] A. H. Guth, Phys. Rev. D 23, 347 (1981); Adv. Ser. Astrophys. Cosmol. 3, 139 (1987).

[10] A. D. Linde, Phys. Lett. 108B, 389 (1982); Adv. Ser. Astrophys. Cosmol. 3, 149 (1987).

[11] A. Albrecht and P. J. Steinhardt, Phys. Rev. Lett. 48, 1220 (1982); Adv. Ser. Astrophys. Cosmol. 3, 158 (1987).

[12] D. Baumann, in Physics of the Large and the Small, TASI 09, Proceedings of the Theoretical Advanced Study Institute in Elementary Particle Physics, Boulder, Colorado, USA, 2009 (World Scientific, 2011), pp. 523-686.

[13] A. D. Linde, Phys. Lett. B 175, 395 (1986).

[14] J. Earman and J. Mosterin, Philos. Sci. 66, 1 (1999).

[15] P. J. Steinhardt, Sci. Am. 304, 36 (2011).

[16] F. Bezrukov, J. Rubio, and M. Shaposhnikov, Phys. Rev. D 92, 083512 (2015).

[17] J. Martin, C. Ringeval, and V. Vennin, Phys. Dark Universe 5-6, 75 (2014).

[18] P. A. R. Ade et al. (Planck Collaboration), Astron. Astrophys. 594, A20 (2016).

[19] P. A. R. Ade et al. (BICEP2 and Keck Array Collaborations), Phys. Rev. Lett. 116, 031302 (2016).

[20] S. Mollerach, Phys. Rev. D 42, 313 (1990).

[21] A. D. Linde and V.F. Mukhanov, Phys. Rev. D 56, R535 (1997).

[22] D. H. Lyth and D. Wands, Phys. Lett. B 524, 5 (2002).
[23] N. Kitajima, D. Langlois, T. Takahashi, and S. Yokoyama, J. Cosmol. Astropart. Phys. 12 (2017) 042.

[24] Z. Trócsányi, Symmetry 12, 107 (2020).

[25] M. Tanabashi et al. (Particle Data Group), Phys. Rev. D 98 , 030001 (2018).

[26] M. E. Peskin and D. W. Schroeder, An Introduction to Quantum Field Theory, 5th ed. (Perseus Books, Reading, MA, 1995).

[27] Z. Péli and Z. Trócsányi, arXiv:1902.02791.

[28] M. Gonderinger, H. Lim, and M. J. Ramsey-Musolf, Phys. Rev. D 86, 043511 (2012).

[29] N. Khan and S. Rakshit, Phys. Rev. D 90, 113008 (2014).

[30] T. Alanne, K. Tuominen, and V. Vaskonen, Nucl. Phys. B889, 692 (2014).

[31] S. Di Chiara, V. Keus, and O. Lebedev, Phys. Lett. B 744, 59 (2015).

[32] M. Duch, B. Grzadkowski, and M. McGarrie, J. High Energy Phys. 09 (2015) 162.

[33] J. Alexander et al., arXiv:1608.08632.

[34] C. T. Byrnes and D. Wands, Phys. Rev. D 74, 043529 (2006).

[35] C. Gordon, D. Wands, B. A. Bassett, and R. Maartens, Phys. Rev. D 63, 023506 (2000).

[36] $R=\delta \sigma H / \dot{\sigma}$ and $S=\delta s H / \dot{\sigma}$.

[37] G. Obied, H. Ooguri, L. Spodyneiko, and C. Vafa, arXiv: 1806.08362.

[38] W. H. Kinney, Phys. Rev. Lett. 122, 081302 (2019).

[39] S. K. Garg and C. Krishnan, J. High Energy Phys. 11 (2019) 075.

[40] This is a particle physics terminology, not to be confused with the scale of inflation $V_{0}^{1 / 4}$. 